\title{
Theoretical Characterization of the reduction Potentials of nucleic acids in solution
}

Valeria D'Annibale, ${ }^{\dagger}$ Alessandro Nicola Nardi, ${ }^{\dagger}$ Andrea Amadei, ${ }^{,, \neq}$and Marco $D^{\prime}$ Abramo*,†

†Department of Chemistry, Sapienza University of Rome, Rome, Italy $\ddagger$ Department of Chemical Sciences and Technology, Tor Vergata University, Rome, Italy

E-mail: andrea.amadei@uniroma2.it; marco.dabramo@uniroma1.it

\section{Supporting Information}




\subsection{Comparison of Vertical Ionization Energies (VIE) as obtained by different DFT functionals}

VIEs of nucleobases have been obtained using different functionals: B3LYP, M06-2X and BP86.

\begin{tabular}{|cc|c|c|}
\hline \multirow{2}{*}{ Base } & VIE $(\mathrm{eV})$ & $\mathrm{VIE}(\mathrm{eV})$ & $\mathrm{VIE}(\mathrm{eV})$ \\
& $\mathrm{B} 3 \mathrm{LYP} / 6-311++\mathrm{G}(2 \mathrm{~d}, 2 \mathrm{p})$ & $\mathrm{M06-2X} / 6-311++\mathrm{G}(2 \mathrm{~d}, 2 \mathrm{p})$ & $\mathrm{BP} 86 / 6-311++\mathrm{G}(2 \mathrm{~d}, 2 \mathrm{p})$ \\
\hline Adenine & 8.21 & 8.48 & 8.85 \\
\hline Guanine & 7.92 & 7.53 & 7.96 \\
\hline Cytosine & 8.71 & 8.96 & 9.29 \\
\hline Thymine & 8.97 & 9.23 & 9.58 \\
\hline
\end{tabular}

\subsection{Excitation Energies}

The Excitation Energies have been evaluated using TDDFT (B3LYP/6-311++G(2d,2p)).

\subsubsection{Excitation Energies for neutral nucleobases}

\begin{tabular}{|cc|c|c|c|}
\hline Exc State & Adenine $(\mathrm{eV})$ & Guanine $(\mathrm{eV})$ & Cytosine $(\mathrm{eV})$ & Thymine $(\mathrm{eV})$ \\
\cline { 2 - 5 } & 4.92 & 4.54 & 4.58 & 4.69 \\
\hline 2 & 4.94 & 4.81 & 4.74 & 4.91 \\
\hline 3 & 5.15 & 4.98 & 5.09 & 5.40 \\
\hline 4 & 5.20 & 5.13 & 5.17 & 5.76 \\
\hline 5 & 5.41 & 5.24 & 5.39 & 5.91 \\
\hline 6 & 5.55 & 5.52 & 5.55 & 6.09 \\
\hline
\end{tabular}

1.2.2 Excitation Energies for radical-cation nucleobases in the equilibrium geometry of neutral nucleobases

\begin{tabular}{|cc|c|c|c|}
\hline Exc. State & Adenine $(\mathrm{eV})$ & Guanine $(\mathrm{eV})$ & Cytosine $(\mathrm{eV})$ & Thymine $(\mathrm{eV})$ \\
\cline { 2 - 5 } & 0.79 & 1.31 & 0.45 & 0.59 \\
\hline 2 & 1.26 & 1.59 & 0.59 & 1.14 \\
\hline 3 & 1.67 & 1.99 & 1.00 & 1.36 \\
\hline 4 & 2.07 & 2.12 & 3.07 & 3.25 \\
\hline 5 & 2.58 & 2.90 & 3.85 & 3.68 \\
\hline 6 & 3.69 & 2.99 & 4.75 & 4.08 \\
\hline
\end{tabular}

1.2.3 Excitation Energies for radical-cations nucleobases in the equilibrium geometry of the relaxed radical-cation nucleobase

\begin{tabular}{|cc|c|c|c|}
\hline Ex. en. State $\mathrm{n}$. & Adenine $(\mathrm{eV})$ & Guanine $(\mathrm{eV})$ & Cytosine $(\mathrm{eV})$ & Thymine $(\mathrm{eV})$ \\
\hline 1 & 1.06 & 1.69 & 0.56 & 1.11 \\
\hline 2 & 1.83 & 2.08 & 0.75 & 1.79 \\
\hline 3 & 2.10 & 2.58 & 1.19 & 1.99 \\
\hline 4 & 2.54 & 2.73 & 3.08 & 3.79 \\
\hline 5 & 2.96 & 3.39 & 4.24 & 4.11 \\
\hline 6 & 4.00 & 3.41 & 5.00 & 4.37 \\
\hline
\end{tabular}


1.3 Standard reduction potentials of deoxynucleosides in water as obtained by PMM procedure and by different theoretical-computational methods reported in literature

\begin{tabular}{|c|c|c|c|c|c|c|}
\hline Base & $\begin{array}{c}V_{\text {red }}(\mathrm{V}) \\
\text { PMM } \\
\text { Deoxy } \\
\text { nucleoside } \\
\end{array}$ & $\begin{array}{c}V_{\text {red }}(\mathrm{V}) \\
\text { Psciuk et al. }^{1} \\
\text { Nucleoside }\end{array}$ & $\begin{array}{c}V_{\text {red }}(\mathrm{V}) \\
\text { Li et al. } \\
\text { Nitrogenous } \\
\text { base }\end{array}$ & $\begin{array}{c}V_{\text {red }}(\mathrm{V}) \\
\text { Paukku, Hill. }{ }^{2} \\
\begin{array}{c}\text { Nitrogenous } \\
\text { base }\end{array}\end{array}$ & $\begin{array}{c}V_{\text {red }}(\mathrm{V}) \\
\text { Wang et al. }{ }^{3} \\
\begin{array}{c}\text { Nitrogenous } \\
\text { base }\end{array}\end{array}$ & $\begin{array}{c}V_{\text {red }}(\mathrm{V}) \\
\text { Wang et al. } \\
\text { Deoxy } \\
\text { nucleoside }\end{array}$ \\
\hline Guanine & 1.05 & 1.21 & 1.44 & 1.49 & 2.03 & 2.08 \\
\hline Adenine & 1.26 & 1.52 & 1.71 & 1.86 & 2.38 & 2.44 \\
\hline Thymine & 1.73 & 1.81 & 1.78 & 2.09 & 2.57 & 2.78 \\
\hline Cytosine & 1.87 & 1.95 & 2.10 & 2.19 & 2.68 & 2.77 \\
\hline
\end{tabular}

1.4 Standard reduction potentials of deoxynucleosides in acetonitrile solution as obtained by PMM procedure and by different theoretical-computational methods reported in literature

\begin{tabular}{|c|c|c|c|c|}
\hline Base & $\begin{array}{l}V_{\text {red }}(\mathrm{V}) \\
\text { PMM } \\
\text { Deoxy } \\
\text { nucleoside }\end{array}$ & $\begin{array}{c}V_{\text {red }}(\mathrm{V}) \\
\text { Crespo-Hernandez et } \\
\text { al. }^{4} \\
\text { Nitrogenous } \\
\text { base }\end{array}$ & $\begin{array}{c}V_{\text {red }}(\mathrm{V}) \\
\text { Crespo-Hernandez et al. }{ }^{4} \\
\text { syn-Deoxynucleoside }\end{array}$ & $\begin{array}{c}V_{\text {red }}(\mathrm{V}) \\
\text { Crespo-Hernandez et al. }{ }^{4} \\
\text { anti-Deoxyucleoside }\end{array}$ \\
\hline Guanine & 1.18 & 2.04 & 2.04 & 1.82 \\
\hline Adenine & 1.50 & 2.20 & 2.17 & 1.99 \\
\hline Thymine & 1.64 & 2.62 & 2.41 & 2.24 \\
\hline Cytosine & 1.79 & 2.44 & 2.34 & 2.12 \\
\hline
\end{tabular}

The standard redox potentials are all reported against the reference standard hydrogen electrode $\left(V_{S H E}=4.281 V\right)$. 


\section{BIBLIOGRAPHY}

1. Psciuk, B. T., Lord, R. L., Munk, B. H. \& Schlegel, H. B. Theoretical determination of one-electron oxidation potentials for nucleic acid bases. in Journal of Chemical Theory and Computation vol. 8 5107-5123 (2012).

2. Paukku, Y. \& Hill, G. Theoretical determination of one-electron redox potentials for DNA bases, base Pairs, and stacks. J. Phys. Chem. A 115, 4804-4810 (2011).

3. Wang, J., Yang, S. \& Zhang, Y. One-electron oxidation and redox potential of nucleobases and deoxyribonucleosides computed by QM/MM simulations. Chem. Phys. Lett. 739, 136948 (2020).

4. Crespo-Hernández, C. E., Close, D. M., Gorb, L. \& Leszczynski, J. Determination of Redox Potentials for the Watson-Crick Base Pairs, DNA Nucleosides, and Relevant Nucleoside Analogues. J. Phys. Chem. B 111, 5386-5395 (2007). 